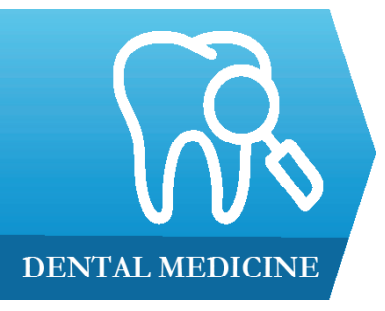

1) Department of Conservative Odontology, Iuliu Hatieganu University of Medicine and Pharmacy, Cluj-Napoca, Romania

2) Department of Restorative Dentistry, Marmara University, Istanbul, Turkey

\title{
The outcome of direct composite restorations using the dental operating microscope
}

\author{
Marius Bud ${ }^{1}$, Mircea Zlăvog ${ }^{1}$, Ștefan Jitaru ${ }^{1}$, Bora Korkut ${ }^{2}$, \\ Sergiu Spataru ${ }^{1}$, Ada Delean ${ }^{1}$
}

\begin{abstract}
Background. The objective of the present study was to compare the outcome of direct composite restorations, with and without the use of additional magnification.

Methods. Twenty extracted molars were selected for the study. Class 1 Black cavities were prepared. All teeth were etched, and bonding agent was applied. Teeth were assigned randomly into two groups of ten each. Group I: restorations were done without the use of magnification, first by placing a layer of SDR $®$ flow + Bulk Fill Flowable on the base of the cavity, and then restoring the morphology with Ceram.X SphereTEC ${ }^{\circledR}$ One. Group II: the same protocol was applied, but using the dental microscope. Teeth were then evaluated before and after finishing the protocol by 5 dental professionals using a series of established criteria. The results were documented and statistically analyzed using the non-parametric Mann-Whitney test.

Results. Statistically significant differences were found when using magnification, before the polishing protocol in "marginal adaptation integrity" and "excess material", and after polishing in "marginal adaptation integrity".

Conclusions. Magnification may be used to increase the quality of the final direct posterior restoration by improving the marginal adaptation integrity, reducing excess material, preventing marginal microleakage, and avoiding subsequent failure.
\end{abstract}

Keywords: direct restoration, magnification, dental operating microscope, restorative dentistry

\section{Introduction}

One of the most common chronic diseases known worldwide is tooth decay, also referred to as dental caries or cavities. Patients are at risk of developing this disease throughout their entire lifetime [1]. Esthetic direct restorations are one of the most preferred treatment methods [2] due to improvements in their mechanical and optical properties and ease in clinical handling [3]. The evolution of composite materials has been continuous since their appearance in the 1960s, with most of the development focusing on the filler technology. This has led to improved wear resistance and has expanded the use of composites to larger posterior restorations [4].

One of the main factors directly associated with the immediate success and lifetime of a restoration is its surface properties. A rough surface will always increase the adhesion of bacteria and dental plaque deposits, making the tooth more susceptible to infiltration and secondary caries [5]. On the other hand, the presence of excess material and a poorly occluding restored tooth not only damages the tooth itself and its associated periodontium, but also harms the dentition of the opposing arch and the overall health of the stomatognathic system [6].

The use of today's new and improved materials has required a corresponding increase in the operator's technical skills. The German physician Saemmish introduced loupes in 1876 to improve visual acuity in the field of 
medicine [7]. Clinical microscopes came later and were introduced in dentistry by Baumann [8]. Dental operating microscopes and loupes have been used by dentists in clinical dentistry for decades, and also by dental technicians to improve the fabrication process of highquality restorations [9]. Studies have shown a reduction in the incidence and severity of musculosketelal disorders in dentists when using magnification systems, because they ensure the optimal vision of the operating field while maintaining an ergonomic position of the body. Moreover, the microscope is proved to lead the highest degree of magnification and the most neutral working position [10].

Working with a microscope allows a more conservative cavity preparation, more precise insertion of restorative materials, and a more precise diagnosis of carious lesions and old restorations that need to be replaced [11]. Magnification may improve the visibility during direct restorative treatments and allows for minimum tooth reduction and a good finishing of the margins [12]. Working with magnification facilitates a precise fitting of matrices and wedges in order to restore a proximal cavity. Shanelec and Tibbets reported that dentists working without magnification can make movements as precise as 1-2 $\mathrm{mm}$. At $20 \times$ magnification, the refinement in movements can be as little as $10-20$ microns at a time. Therefore, the limitation regarding the precision of treatment is not in the hands, but in the eyes [13].

Although dental operating microscopes and other magnification devices are known for offering the operator an ergonomic posture and additional comfort and control [14], their potential to improve the outcome of direct restorations is still unclear and has not yet been reported in studies.

The objective of the present study was to compare the outcome of occlusal direct composite restorations, with and without the use of additional magnification.

\section{Methods}

Twenty maxillary and mandibular molars, extracted for periodontal or surgical reasons and free of restorations or existing caries, were selected for the study. The teeth were stored in artificial saliva at 37 degrees temperature and cleaned before use with an Air-flow Handy 2+ and a Powder Clasic Confort (EMS Company, Nyon, Switzerland). Extended occlusal Class I Black cavities were prepared using a high speed handpiece (NSK Company, Japan) with water spray coolant. All cavities were prepared using a straight cylinder diamond burr (Kerr Company, Germany). The completed preparations had $2 \mathrm{~mm}$ of healthy marginal ridge remaining, half of each cusp, and an occlusal depth of minimum $2 \mathrm{~mm}$. The measurements were done using a periodontal probe. After completion of the preparations, the teeth were thoroughly rinsed with water to remove debris and dried with air. Teeth were then assigned randomly into two groups $(n=10)$ :
Group I - Direct composite restorations without the use of magnification and Group II - Direct composite restorations using magnification.

Group I: After completion of the preparations, 36\% o-phosphoric acid (Blue Etch, Cerkamed, Poland) was applied on the enamel for 15 seconds. After 15 seconds the etchant was applied on dentin, for a total etching time of 30 seconds for enamel and 15 seconds for dentin. The cavity was then thoroughly rinsed with water for 20 seconds and gently air-dried. A bonding agent (Xp Bond ${ }^{\mathrm{TM}}$, Dentsply Sirona, Germany) was applied according to the manufacturer's instructions. First, a layer of bulk (SDR ${ }^{\circledR}$ Bulk Fill Flowable, Dentsply Sirona) was placed on the base of the cavity, leaving approximately $1.5 \mathrm{~mm}$ occlusal space. After lightcuring, the successive cusp buildup technique was used with Ceram.X SphereTEC $\AA$ One (Dentsply Sirona) to restore the morphology. Cusps were restored one at a time, up to the level of the occlusal enamel using LM-Arte Set (LM Dental, Finland) and a common composite brush (Enamel Plus C, Micerium Company, Italy). Each cusp was light-cured for 5 seconds from an occlusal direction and the final restoration was light cured for 20 seconds more. The choice of materials was based on their wide usage in clinical practice for direct posterior restorations and qualities proven in the current literature [15].

Group II: Following cavity preparation teeth were placed under the surgical microscope (OMS 2350, Zumax Company, China) using 6.9x magnification, a level suitable for detecting and avoiding compromising bio-mechanical factors when preparing direct composite restorations [16]. An orange light filter was used in order to prevent light curing the composite during working. The same restoration protocol was used as in Group I. Each tooth was restored within 30 minutes, at elbow height, corresponding to the average position in the dental chair.

All teeth were restored by the same operator and were randomly evaluated by five dentists with more than 3 years experience. The 5 clinicians were calibrated for evaluation of the restorations prior to starting the study. Samples were examined under the microscope, with the same magnification conditions (OMS 2350, Zumax Company, China) using 6.9x magnification and a sharp dental explorer. Each restoration was clinically examined independently, with the help of a dental assistant that recorded the results. Four parameters including "marginal adaptation integrity", "excess material", "morphology accuracy", and "surface defects" were assessed. For each criterion, the evaluators were asked to give a score from 1 to 5 using "Table I" as a guideline. For the evaluation guideline, a modified version of the "Ryge Criteria for direct evaluation" was used as model and was adapted to better suit the authors preference [17]. All restorations were evaluated before and after polishing in order to check whether certain criteria were influenced by the polishing step. 
Table I. Evaluation guideline.

\begin{tabular}{|c|c|c|c|c|c|}
\hline \multirow{2}{*}{ Parameter } & \multicolumn{5}{|c|}{ Score } \\
\hline & 5 & 4 & 3 & 2 & 1 \\
\hline $\begin{array}{l}\text { Marginal Adaptation } \\
\text { Integrity }\end{array}$ & $\begin{array}{c}\text { Explorer does not catch when } \\
\text { drawn across the restoration/tooth } \\
\text { interface (no voids) }\end{array}$ & $<>$ & $\begin{array}{l}\text { Explorer catches on less than } \\
1 / 2 \text { of the restoration contour }\end{array}$ & $>$ & $\begin{array}{l}\text { Explorer catches on more than } \\
1 / 2 \text { of the restoration contour }\end{array}$ \\
\hline Excess Material & $\begin{array}{l}\text { No excess material present } \\
\text { outside the restoration }\end{array}$ & $<>$ & $\begin{array}{c}\text { Excess material present on } \\
\text { less than } 1 / 2 \text { of the restoration } \\
\text { contour }\end{array}$ & $\diamond$ & $\begin{array}{c}\text { Excess material present on } \\
\text { more than } 1 / 2 \text { of the restoration } \\
\text { contour }\end{array}$ \\
\hline $\begin{array}{l}\text { Morphology } \\
\text { Accuracy }\end{array}$ & $\begin{array}{l}\text { Restoration morphology } \\
\text { accurately respects/continues the } \\
\text { tooth morphology }\end{array}$ & $<>$ & $\begin{array}{l}\text { Restoration morphology } \\
\text { partially respects/continues } \\
\text { the tooth morphology }\end{array}$ & $<$ & $\begin{array}{l}\text { Restoration morphology } \\
\text { doesn't respect/continue the } \\
\text { tooth morphology }\end{array}$ \\
\hline Surface Defects & $\begin{array}{l}\text { The restoration doesn't have } \\
\text { surface defects (gaps, missing } \\
\text { material on the surface) }\end{array}$ & $<>$ & $\begin{array}{c}\text { The restoration has minimal } \\
\text { defects (1-2 gaps on the } \\
\text { surface) }\end{array}$ & $\diamond$ & $\begin{array}{l}\text { The restoration has major } \\
\text { surface defects }\end{array}$ \\
\hline
\end{tabular}

After the first evaluation, the finishing and polishing were done using a finishing carbide flame burr (BWFG7104; Kerr Company, Germany), followed by silicone rubbers (Kenda, Vaduz, Liechtenstein) and Prisma Gloss Polishing Paste (Dentsply Sirona, Germany). All teeth were then examined a second time using the same criteria.

The data were analyzed using SPSS version 20 (SPSS, IBM Corp., Armonk, NY) using the non-parametric Mann-Whitney test at the $\mathrm{p}<.05$ significance level, followed by a two-way mixed effects, absolute agreement, intraclass correlation coefficient (ICC) used to determine the inter-rater reliability.

\section{Results}

A general improvement in scores was observed in favor of using magnification both before and after the polishing step. The median global scores of the evaluated specimens after direct restoration with the naked eye and with microscope magnification are shown in figure 1.

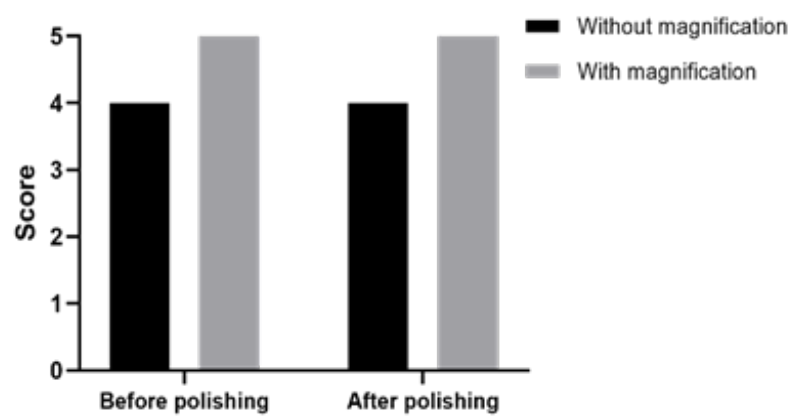

Figure 1. Median global scores pre/post polishing.

Statistically significant differences were found when using magnification, before the polishing protocol in "marginal adaptation integrity" (with the lowest score being 1 for Group I and 3 for Group II) and "excess material" (lowest score 1 for Group I and 2 for Group II).

After the polishing protocol, statistically significant differences were found for "marginal adaptation integrity" (with the lowest score being 2 for Group I, and 4 for Group II). No significant differences were found for the "morphology accuracy" and "surface defects" criteria in any evaluation, both before and after polishing. Cumulated median representations of each evaluated criterion after the polishing protocol are shown in figures 2, 3, 4 and 5 .

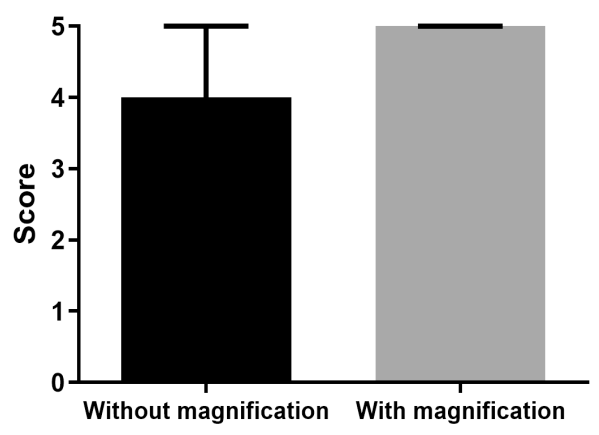

Figure 2. Marginal adaptation integrity after polishing (all ev., $\mathrm{p}<0.001)$.

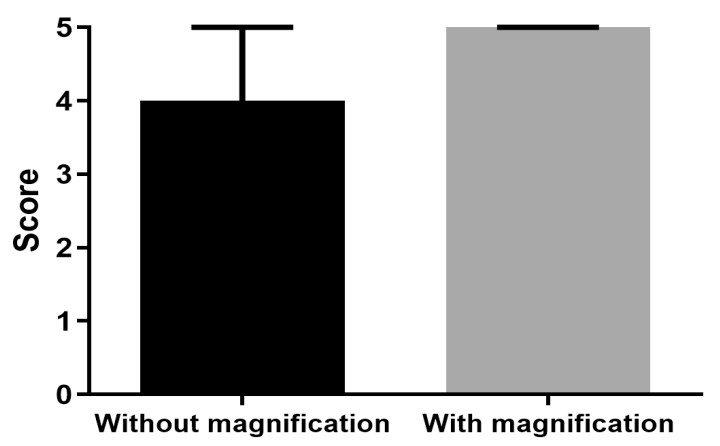

Figure 3. Excess material after polishing (all ev., $\mathrm{p}<0.01$ ). 


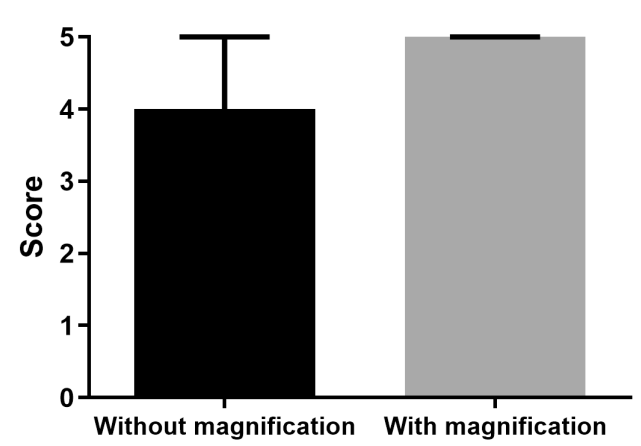

Figure 4. Morphology accuracy after polishing (all ev., $\mathrm{p}<0.01$ ).

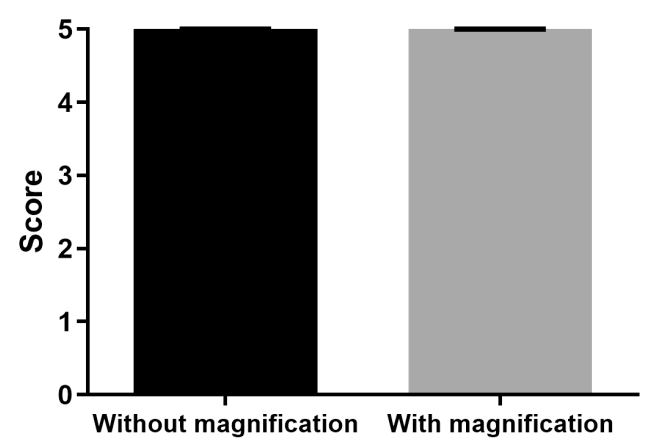

Figure 5. Surface defects after polishing (all ev., $\mathrm{p}<0.05$ ).

The Intraclass correlation coefficient based on the $95 \%$ confident interval showed a result of .640 which indicates a moderate agreement between the raters [18].

\section{Discussion}

For this study, twenty extracted molars with class I Black cavities prepared were directly restored with composite using unaided eye vision (Group 1) and using microscope magnification (Group 2) and then evaluated by 5 calibrated observers. The results showed that the use of microscope magnification may significantly improve the outcome of direct restorations for some of the evaluated criteria like the marginal integrity, both before and after the polishing step, or the excess composite overhangs before the polishing step. However, no differences were found regarding the occlusal morphology and surface defects criteria when magnification was used. We may stipulate that using a microscope leads to improvement in small important details of the procedure directly related to the restoration's long term success, like the marginal integrity.

The first evaluated criterion in our study was the marginal adaptation integrity or the absence of voids between composite and enamel, which are clinically related to microleakage. Microleakage is one of the most frequently encountered problems in restorative dentistry [19], leading to recurrent and secondary caries, postoperative sensitivity, enamel fracture, and marginal deterioration [20]. Significant improvement was found in the marginal adaptation integrity, both before and after the polishing step, when using the microscope $(\mathrm{p}<0.001)$. These results have strong clinical importance related to the recommendation to use magnification in order to increase the outcome of direct restorations.

When the excess of material was evaluated, we noticed a significant improvement when magnification was used before the finishing and polishing step $(p<0.001)$. The difference was not significant after the finishing and polishing were done $(\mathrm{p}>0.05)$. For the direct restorations done with the naked eye, the finishing and polishing step, if well done, may remove the composite excess in order to obtain the same filling quality as when using the microscope. We may stipulate so far that the removal of composite excess by finishing and polishing is not improved by the use of magnification. Similar results were found when removing composite around orthodontic brackets; the operating microscope performed very similarly to the removal of conventional composite with the naked eye [21].

The excess composite that extends beyond the preparation margin is essentially a thin flap that overlies the tooth surface. Food and debris may collect underneath the flap, eventually causing carious or non-carious stains under it. The dentist may be able to use the microscope for easier detection, and polish away the flap [16]. The use of magnification before polishing showed improvement in regards to excess material which may shorten the amount of time needed for the finishing protocol. Less excess material will also shorten the time needed for occlusal adjustments.

When observers evaluated the morphology accuracy and surface defects of the direct restorations, obtained with and without microscope magnification, no significant differences were found $(\mathrm{p}>0.05)$. That means that magnification doesn't seem to help in improving occlusal morphology or in avoiding composite surface defects. This fact may come in favor of the majority of dental practitioners who do not include the use of magnification in their daily routine when doing direct posterior restorations. However, it may be inaccurate to state that the operating microscope does not add precision to the composite modeling step. In order to investigate this, new studies without any time restriction could deliver more relevant data, as this might have contributed to the poor performance of the dental operating microscope. No matter how experienced the operator may be, a longer modeling time will usually be necessary whenever using a dental operating microscope due to a more detailed vision. Also, using the orange filter to prevent light-curing 
may impede depth vision and make the modelling of the composite material more difficult. Nevertheless, Leknius and Geissberger reported that procedural errors decrease significantly when working with magnification [22].

A limitation of this study is that the evaluation included only criteria related to the surface of the restoration, aspects that can be clinically observed. The use of high magnification may improve many other elements which cannot be evaluated on the composite surface and therefore they were skipped. Some of these may be:

- removing excessive adhesives [23];

- verifying that the tip of a micro-brush is thin enough in diameter to penetrate all aspects of the preparation, and if the bonding was correctly applied on all surfaces of the cavity;

- checking if the composite is flowing into all aspects of the preparation, in all irregularities;

- detecting microscopic air bubbles in flowable composite, located either inside or on the external surface or margin of the flowable composite bolus;

- conservative tooth preparation [24];

- improvement of the working posture using magnification [25];

- detecting impurities that may appear in materials [16].

Although the efficacy of using the microscope is not yet clear for certain treatment steps, the improvement in marginal integrity that implicitly lowers the chances of secondary caries is a strong reason to recommend using it. Even considering limitations of this study, we can conclude that the use of the microscope for direct esthetic posterior restorations leads to an immediate increase in the quality of treatment with implications for long-term success.

\section{Conclusions}

Magnification may be used to increase the quality of the final direct posterior restoration by improving the marginal adaptation integrity, reducing excess material, preventing marginal microleakage, and avoiding subsequent failure.

\section{Acknowledgements}

We would like to thank Andrei Mitre for his help with statistical advice and contribution to this manuscript.

\section{References}

1. Selwitz RH, Ismail AI, Pitts NB. Dental caries. Lancet. 2007;369:51-59.

2. Demarco FF, Collares K, Correa MB, Cenci MS, Moraes RR, Opdam NJ. Should my composite restorations last forever?
Why are they failing? Braz Oral Res. 2017;31(suppl 1):e56.

3. Meenakumari C, Bhat KM, Bansal R, Singh N. Evaluation of mechanical properties of newer nanoposterior restorative resin composites: An in vitro study. Contemp Clin Dent. 2018;9(Suppl 1):S142-S146.

4. Fugolin APP, Pfeifer CS. New Resins for Dental Composites. J Dent Res. 2017;96:1085-1091.

5. Salerno M, Loria P, Matarazzo G, Tomè F, Diaspro A, Eggenhöffner R. Surface morphology and tooth adhesion of a novel nanostructured dental restorative composite. Materials (Basel). 2016;9:203.

6. Davies S, Gray RM. What is occlusion? Br Dent J. 2001;191:235-238, 241-245.

7. Shanelec DA. Optical principles of loupes. J Calif Dent Assoc. 1992;20:25-32.

8. Baumann RR. What is the use of the microscope for the dentist? Quintessenz. 1975;26:33-34.

9. Winter RR, Cornell DF, Vingoren GJ, Patrick RB. Use of magnification in dental technology. J Esthet Restor Dent. 2003;15:409-415; discussion 416.

10. Valachi B, Valachi K. Preventing musculoskeletal disorders in clinical dentistry: strategies to address the mechanisms leading to musculoskeletal disorders. J Am Dent Assoc. 2003;134:1604-1612.

11. Moura JR Jr. Operating Microscopes in Restorative Dentistry: the Pursuit of Excellence. Int Dent. 2009;10:411. Available from: http://www.moderndentistrymedia. com/sept_oct2008/moura.pdf

12. Sheets CG. The periodontal-restorative interface: enhancement through magnification. Pract Periodontics Aesthet Dent. 1999;11:925-931; quiz 932.

13. Tibbetts LS, Shanelec D. Periodontal microsurgery. Dent Clin North Am. 1998;42:339-359.

14. Caplan SA. Magnification in dentistry. J Esthet Dent. 1990;2:17-21.

15. Mahmoud SH, El-Embaby AE, AbdAllah AM. Clinical performance of ormocer, nanofilled, and nanoceramic resin composites in Class I and Class II restorations: a three-year evaluation. Oper Dent. 2014;39:32-42.

16. Mamoun J. Preparing and Restoring Composite Resin Restorations. The Advantage of High Magnification Loupes or the Dental Surgical Operating Microscope. N Y State Dent J. 2015;81:18-23.

17. Barnes DM, Blank LW, Gingell JC, Gilner PP. A clinical evaluation of a resin-modified. Glass ionomer restorative material. J Am Dent Assoc. 1995;126:1245-1253.

18. Koo TK, Li MY. A Guideline of Selecting and Reporting Intraclass Correlation Coefficients for Reliability Research. J Chiropr Med. 2016;15:155-163.

19. Ozel E, Soyman M. Effect of fiber nets, application techniques and flowable composites on microleakage and the effect of fiber nets on polymerization shrinkage in class II MOD cavities. Oper Dent. 2009;34:174-180.

20. Radhika MR, Sajjan GS, Kumaraswamy BN, Mittal N. Effect of different placement techniques on marginal 
microleakage of deep class-II cavities restored with two composite resin formulations. J Conserv Dent. 2010;13:915.

21. Alencar EQ, Nobrega ML, Dametto FR, Santos PB, Pinheiro FH. Comparison of two methods of visual magnification for removal of adhesive flash during bracket placement using two types of orthodontic bonding agents. Dental Press J Orthod. 2016;21:43-50.

22. Leknius $\mathrm{C}$, Geissberger $\mathrm{M}$. The effect of magnification on the performance of fixed prosthodontic procedures. J Calif
Dent Assoc. 1995;23:66-70.

23. van As GA. The use of extreme magnification in fixed prosthodontics. Dent Today. 2003;22:93-99.

24. Bud M, Jitaru S, Lucaciu O, Korkut B, Dumitrascu-Timis $\mathrm{L}$, Ionescu C, Cimpean S, Delean A. The advantages of the dental operative microscope in restorative dentistry. Med Pharm Rep. 2021;94:22-27.

25. Bud M, Pricope R, Pop RC, Onaca R, Swerts PJ, Lucaciu $\mathrm{O}$, Delean A. Comparative analysis of preclinical dental students' working postures using dental loupes and dental operating microscope. Eur J Dent Educ. 2021;25:516-523. 